\title{
ARC- AND TREE-PRESERVING TRANSFORMATIONS
}

\author{
BY \\ D. W. HALL $\left({ }^{1}\right)$ AND G. T. WHYBURN
}

1. Introduction. In an earlier paper $\left({ }^{2}\right)$ by one of us, referred to hereafter as A.P.T., arc-preserving transformations were defined and studied in connection with an irreducibility condition on the transformation. It was shown, for example, that if $A$ and $B$ are compact locally connected metric continua which are cyclic (that is, without cut points) any single valued continuous arc-preserving and irreducible transformation $T(A)=B$ of $A$ on to $B$ is necessarily a homeomorphism. ("Arc-preserving" means that the image of every simple arc in $A$ is either a simple arc or a single point of $B$; irreducibility of $T$ means that no proper subcontinuum of $A$ maps onto all of $B$.) It was shown, furthermore, that in case $A$ is hereditarily locally connected the same conclusion holds without the assumption of irreducibility; and the prediction was made that this is true in the general case.

Now as pointed out in A.P.T., if $A$ is a compact continuum and $T(A)=B$ is continuous, then, since the property of being a subcontinuum of $A$ mapping onto all of $B$ under $T$ is inducible, there always exists a subcontinuum $A_{1}$ of $A$ such that $T\left(A_{1}\right)=B$ and $T$ is irreducible on $A_{1}$. However, since local connectedness of $A$ would certainly not in general insure local connectedness of $A_{1}$, it is not possible always to reduce the set $A$ so as to make the transformation irreducible without sacrificing essential properties of $A$.

In the present paper we propose not only to completely justify the earlier prediction referred to above, but also to obtain theorems concerning a much more general type of transformation than "arc-preserving" which will give all the theorems of the first three sections of A.P.T. as immediate corollaries.

R. G. Simond $\left({ }^{3}\right)$ has studied tree-preserving transformations on locally connected compact and metric continua (that is, transformations $T(A)=B$ satisfying the condition that the image of every tree (or dendrite) in $A$ is a tree in $B$ ). Miss Simond has proved with considerable difficulty that every arc-preserving transformation is tree-preserving. We show that this result is an immediate consequence of one of our theorems, as it is also of a theorem of A.P.T. In fact our first principal result, the proof of which is very much simpler than that given by Simond, shows that in order that $T(A)=B$ be tree-preserving it is necessary and sufficient that the image of every simple

Presented to the Society, December 28, 1939; received by the editors January 8, 1940.

(1) This work was started when the first named author was a National Research Fellow at the University of Virginia.

(2) See G. T. Whyburn, Arc-preserving transformations, American Journal of Mathematics, vol. 58 (1936), pp. 305-312.

(3) Duke Mathematical Journal, vol. 4 (1938), pp. 575-589. 
arc in $A$ shall be a tree in $B$. We also give an independent proof of the Simond theorem.

If $A$ is a tree, every simple arc in $A$ is a cyclic chain $\left(^{4}\right)$ in $A$. This shows at once that if $A$ and $B$ are both trees and $T(A)=B$ is continuous, then in order that $T$ be arc-preserving it is necessary and sufficient that $T$ be cyclic chain-preserving. This immediately suggests another of our main results: If $A$ is a compact locally connected continuum and $T(A)=B$ is continuous, then in order that $T$ be arc-preserving it is necessary and sufficient that $T$ be both tree-preserving and cyclic chain-preserving. We give this theorem added meaning by obtaining a somewhat unexpected characterization of tree-preserving transformations in terms of the action of these transformations and their inverses on the sets $A$ and $B$.

In $\$ 5$ a characterization of $A$-set reversing transformations $\left(^{5}\right)$ is given which supplements the treatment of this type of transformation initiated in A.P.T.

In conclusion we might mention that if $B$ is cyclic, the following types of transformations are equivalent: (a) arc-preserving, (b) tree-preserving, (c) $A$ set reversing, (d) monotone retracting.

Throughout the paper all transformations are assumed to be single valued and continuous and all continua compact and metric.

2. Principal results. We assume throughout this section that $A$ and $B$ are locally connected continua and that $T(A)=B$ maps every arc of $A$ onto a dendrite in $B$.

\section{(2.0) The image of every dendritic graph in $A$ is a dendrite in $B$.}

Proof. (By induction on the number of end points.) If the number of end points in the graph is 2 , then the hypothesis gives the conclusion since the graph is an arc. Suppose that any dendrite in $A$ having $k$ or less end points $(k>0)$ maps onto a dendrite in $B$. Let $D$ be a dendrite in $A$ having $k+1$ end points. Let $p$ be a branch point in $D$ giving a decomposition $D=D_{1}+D_{2}+D_{3}$, where $D_{1}, D_{2}$, and $D_{3}$ are dendrites intersecting by pairs in $p$. Since $D_{1}, D_{2}, D_{3}$, $D_{1}+D_{2}, D_{1}+D_{3}, D_{2}+D_{3}$ are dendrites having at most $k$ end points, each of their transforms is a tree. Hence $T\left(D_{1}\right) \cdot T\left(D_{3}\right)$ and $T\left(D_{2}\right) \cdot T\left(D_{3}\right)$ are con-

(4) If $M$ is a locally connected compact and metric continuum and $A$ is a closed subset of $M$ containing every simple $\operatorname{arc} a x b$ of $M$ such that $a$ and $b$ are points of $A$, then $A$ is called an $A$-set. By the cyclic chain in $M$ determined by two points $a$ and $b$ of $M$ and designated by $C(a, b)$ is meant the product of all $A$-sets in $M$ containing both $a$ and $b$. It is the minimal $A$-set in $M$ containing both these points. The cyclic chains in $M$ are closely related to the decomposition of $M$ into its cyclic elements, for which see Kuratowski and Whyburn, Fundamenta Mathematicae, vol. 16 (1930), pp. 305-331. See also G. T. Whyburn, American Journal of Mathematics, vol. 50 (1928), pp. 167-194, and W. L. Ayres, these Transactions, vol. 30 (1928), pp. 567-578, and vol. 31 (1929), pp. 595-695.

$\left(^{5}\right)$ For definition, see $\$ 5$. 
nected; and since both of these sets contain $T(p)$, their sum $T\left(D_{3}\right) \cdot T\left(D_{1}+D_{2}\right)$ is connected. Thus $T(D)=T\left(D_{1}+D_{2}+D_{3}\right)$ is a dendrite.

(2.1) If $B$ is cyclic, $T$ is $A$-set reversing.

Proof. Otherwise there exists a simple arc $b_{1} x b_{2}$ in $A$ such that $T\left(b_{1}\right)$ $=T\left(b_{2}\right)=b$, but $T^{-1}(b) \cdot b_{1} x b_{2}=b_{1}+b_{2}$. Let $T\left(b_{1} x b_{2}\right)=X$ and $K=T^{-1}(X)$. From (2.0) it follows that $X$ is a dendrite; hence $X \neq B$. Thus $A-K \neq 0$.

(i) For any component $R$ of $A-K, T(F(R))$ is a single point $\left(^{6}\right)$.

Otherwise there exists a simple arc $c y d$ in $R+c+d$ such that $c$ and $d$ lie in $F(R)$ and $T(c) \neq T(d)$. But now if both $c$ and $d$ are on $b^{\prime} x b^{\prime \prime}$ (in the order $\left.b^{\prime}, c, d, b^{\prime \prime}\right)$ we let $t=b^{\prime} c+c y d+d b^{\prime \prime}$; if not let $t$ be a dendritic graph in $A$ which contains both $b_{1} x b_{2}$ and $c y d$. This is impossible by (2.0) since in either case $T(t)$ must contain a simple closed curve.

(ii) There exist two components $R$ and $S$ of $A-K$ such that $a=T(F(R))$ $\neq T(F(S))=c$ and $T(R) \cdot T(S) \neq 0$.

For let $R$ be any component of $A-K$ and let $Q$ be the sum of all those components $U$ of $A-K$ such that $T(F(U))=T(F(R))=a$. Then since $Q+T^{-1}(a)$ is closed, it follows that $T(Q)+a$ is closed. Since $a$ is not a cut point of $B$, it follows that some point $x$ of $T(Q)$ must be a limit point of $B-T(Q)$. (We know that $T(Q)+a \neq B$ since $X$ is not a single point.) But $Q$ is open in $A$; consequently $T^{-1}(x)$ must intersect some component $S$ of $A-K$ which does not belong to $Q$. Thus if we set $c=T(F(S)$ ), (ii) is satisfied.

Now to prove (2.1), let $y^{\prime}$ and $y^{\prime \prime}$ be points of $R$ and $S$, respectively, such that $T\left(y^{\prime}\right)=T\left(y^{\prime \prime}\right)=y$. Then there exists a dendritic graph $t^{\prime}$ in $A$ containing $y^{\prime}$ and $b_{1} x b_{2}$. If $t^{\prime}$ contains $y^{\prime \prime}$, let $t=t^{\prime}$. Otherwise there exists a dendritic graph $t$ in $A$ containing both $y^{\prime \prime}$ and $t^{\prime}$. It is immediate that $T(t)$ contains a simple closed curve, contradicting (2.0).

We have at once:

(2.11) If $A$ and $B$ are both cyclic, $T$ is a homeomorphism.

(2.2) In order that a single valued continuous transformation $T(A)=B$ shall be tree-preserving it is necessary and sufficient that the image of every arc in $A$ shall be a tree in $B$.

Proof. The necessity is trivial. To prove the sufficiency suppose $A$ is a tree and that $B$ has a true cyclic element $E_{b}$. Let $W(B)=E_{b}$ be monotone and retracting. Then $W T(A)=E_{b}$ is a transformation whïch maps arcs into trees. Thus, by (2.1), $W T$ is $A$-set reversing, hence monotone. But this makes $E_{b}$ a tree, which is absurd.

( $\left.{ }^{(}\right)$For any open set $G, F(G)$ denotes the boundary of $G$, that is, the set $\bar{G}-G$. 
(2.3) If $B$ is cyclic and no $A$-set in $A$ other than $A$ itself maps onto all of $B$, then $A$ is cyclic and $T$ is a homeomorphism.

Proof. For if $A$ had a cut point $p$, we could write $A=A_{1}+A_{2}$, where $A_{1}$ and $A_{2}$ are $A$-sets with $A_{1} \cdot A_{2}=p$. Then since, by (2.1), $T$ is monotone, either $A_{1}-p$ or $A_{2}-p$, say $A_{1}-p$, contains the set $T^{-1}(B-T(p))$, as this latter set is connected. But this gives $T\left(A_{1}\right)=B$.

(2.4) If $B$ is cyclic, $T$ is equivalent to a monotone transformation retracting $A$ onto some true cyclic element of $A$. Thus, in this situation, "arc-preserving," "tree-preserving," "A-set reversing," and "monotone retracting" are all equivalent.

Proof. For let $E_{a}$ be a minimal $A$-set in $A$ mapping onto all of $B$ under $T$. Then since $T\left(E_{a}\right)=B$ is a transformation mapping arcs into trees, it follows from (2.3) that $E_{a}$ is a cyclic element of $A$ and $E_{a}$ maps onto all of $B$ topologically under $T$. Thus if for each $y$ in $B$ we set $h(y)=E_{a} \cdot T^{-1}(y)$, then $h$ is topological and the transformation $h T(A)=E_{a}$ is retracting. Furthermore, $h T$ is monotone since both $h$ and $T$ are monotone. Obviously $h T$ is equivalent to $T$, since $h^{-1}(h T)=T$.

Definition. For any true cyclic element $E_{a}$ of $A$ we define $E_{a}^{0}$ as the set of all internal points of $E_{a}$, that is, all points $x$ of $E_{a}$ which are non-cut points of $A$. It is well known that the set of all non-internal points of any such $E_{a}$ is countable.

(2.5) For each true cyclic element $E_{b}$ of $B$ there exists a unique true cyclic element $E_{a}$ of $A$ such that $T\left(E_{a}\right)=E_{b}$. The transformation $T$ is a homeomorphism on $E_{a}$ and $T^{-1}$ is single valued on $T\left(E_{a}^{0}\right)$.

Proof. For let $W(B)=E_{b}$ be monotone and retracting. Since $W T(A)=E_{b}$ is a transformation which maps arcs in to trees, it follows from (2.4) that there exists a true cyclic element $E_{a}$ of $A$ and a homeomorphism $h\left(E_{b}\right)=E_{a}$ such that $h W T(A)=E_{a}$ is monotone and retracting. Since $E_{a}$ maps topologically under $h W T$, it must therefore map topologically under $T$. Let $y=T(x)$ be a point of $T\left(E_{a}^{0}\right)$, where $x$ lies in $E_{a}^{0}$. Since $x$ is an internal point of $E_{a}$ and $h W T$ is monotone and retracting, we see that $x=(h W T)^{-1}(x)=T^{-1} W^{-1} h^{-1}(x)$ $=T^{-1} W^{-1}(y) \supset T^{-1}(y)$, and hence $x=T^{-1}(y)$. The uniqueness of $E_{a}$ follows at once from this single-valuedness of $T^{-1}$.

(2.6) Let $A$ be a compact locally connected continuum and $T(A)=B$ be continuous. Then in order that $T$ be tree-preserving it is necessary and sufficient that for each true cyclic element $E_{b}$ of $B$ there exist a true cyclic element $E_{a}$ of $A$ mapping onto $E_{b}$ topologically under $T$ and such that $T^{-1}$ is single valued on the set $T\left(E_{a}^{0}\right)$.

Proof. The necessity follows from (2.5). To establish the sufficiency we need only, in virtue of (2.2), show that the image of every simple arc $t$ in $A$ 
is a tree in $B$. Assuming the contrary, $T(t)$ must contain a simple closed curve $J^{\prime}$. Let $E_{b}$ be the true cyclic element of $B$ containing $J^{\prime}$ and $E_{a}$ the true cyclic element of $A$ which satisfies the conditions of the theorem. It follows at once that $t \cdot E_{a}$ is a simple arc $a x b$ which maps into an arc $a^{\prime} x^{\prime} b^{\prime}$ of $J^{\prime}$. Let $a^{\prime} y^{\prime} b^{\prime}$ be the other arc of $J^{\prime}$, and suppose that $y^{\prime}$ is the image of an internal point of $E_{a}$. Then $T^{-1}\left(y^{\prime}\right)$ contains a point of $E_{a}$ and a point of $t-E_{a}$, which is impossible.

(2.7) Let $A$ be a compact locally connected continuum and let $T(A)=B$ be continuous. Then in order that $T$ be arc-preserving it is necessary and sufficient that it be tree-preserving and that the image of each cyclic chain $\left({ }^{7}\right)$ in $A$ be a cyclic chain in $B$.

Proof. Necessity: The first condition is necessary by (2.2). That the second condition is necessary results essentially from the fact that, for arc-preserving transformations, (1) $A$-sets map onto $A$-sets, and (2) the property of having any three points on an arc is invariant.

We first show that $A$-sets map onto $A$-sets. Let $A^{\prime}$ be an $A$-set in $A$ and $T\left(A^{\prime}\right)=B^{\prime}$. For any cyclic element $E_{b}$ of $B$ intersecting $B^{\prime}$ in at least two points, let $E_{a}$ be the corresponding cyclic element of $A$ given by (2.6). Since $E_{b} \cdot B^{\prime}$ is a nondegenerate continuum and $T\left(E_{a}\right)=E_{b}$ is topological, $E_{b} \cdot B^{\prime}$ must contain the image $y$ of at least one internal point $x$ of $E_{a}$. Then since $x=T^{-1}(y)$ we see that $x$ must lie in $A^{\prime}$. Thus $E_{a}$ is contained in $A^{\prime}$, consequently $E_{b}$ is contained in $B^{\prime}$. Therefore, $B^{\prime}$ is an $A$-set in $B$.

Now to prove the necessity of the second condition of the theorem, let $C(a, b)$ be a cyclic chain in $A$. Then $T(C(a, b))=K$ is an $A$-set in $B$. Let $x, y, z$ be points of $K$ and $x^{\prime}, y^{\prime}, z^{\prime}$ be points of $C(a, b) \cdot T^{-1}(x), C(a, b) \cdot T^{-1}(y)$, $C(a, b) \cdot T^{-1}(z)$, respectively. There exists an arc $c d$ in $C(a, b)$ containing $x^{\prime}, y^{\prime}$, and $z^{\prime}$. Hence $T(c d)$ is an arc in $K$ containing $x, y$, and $z$. Therefore, $K$ is a cyclic chain (since for $A$-sets the property of being a cyclic chain is equivalent to the property of containing an arc through any three points).

Sufficiency: Let $a b$ be any simple arc in $A$. We first show that if $E_{b}$ is any true cyclic element of $B$ such that $E_{b} \cdot T(a b)$ is nondegenerate and $E_{a}$ is the corresponding cyclic element of $A$ given by (2.6) and $x y$ is the arc $E_{a} \cdot a b$, then $T(a x+y b) \cdot E_{b}=x+y$. (We may suppose the order $a, x, y, b$.) If this is not so, then $T(a x) \cdot E_{b}$ or $T(y b) \cdot E_{b}$, say $T(a x) \cdot E_{b}$, is a nondegenerate continuum; hence there must exist a point $z$ distinct from $T(x)$ of $T\left(E_{a}^{0}\right)$ which belongs to $E_{b} \cdot T(a x)$. This contradicts (2.6), since $T^{-1}(z)$ intersects both $E_{a}$ and $a x-x$. Thus $E_{b} \cdot T(a b)$ is a simple arc $x^{\prime} y^{\prime}=T(x y)$. Furthermore, no interior point of $x^{\prime} y^{\prime}$ is a limit point of $T(a b)-x^{\prime} y^{\prime}$.

Now if $T(a b)$ is not a simple arc it cannot be a simple closed curve. This follows either from (2.2) or from what was just shown. Hence $T(a b)$ must con-

$\left({ }^{7}\right)$ See footnote 4. 
tain a triod $o c+o d+o e=t$. But, since $T$ is cyclic chain-preserving, $T(C(a, b))$ must contain a simple arc through the three points $c, d$, e, say $c d e$. Then either $c d$ or $d e$ does not contain $o$, say $d e$ does not contain $o$. Thus $o d+o e+d e$ contains a simple closed curve $J_{b}$ containing nondegenerate subarcs $o d^{\prime}$ and $o e^{\prime}$ of $o d$ and $o e$; and if $E_{b}$ is the cyclic element of $B$ containing $J_{b}, E_{b} \cdot T(a b)$ $\supset E_{b} \cdot t \supset d^{\prime} e^{\prime}=o d^{\prime}+o e^{\prime}$ and $o$ is a limit point of $T(a b)-d^{\prime} e^{\prime}$ contrary to what was shown above.

3. Supplementary results. We give here some additional results, throwing light on the action of arc-preserving transformations. We assume throughout this section that $A$ is a compact locally connected continuum and that $T(A)=B$ is arc-preserving.

(3.1) The image of each $A$-set in $A$ is an $A$-set in $B$; the image of each cyclic chain in $A$ is a cyclic chain in $B$.

(3.2) For each true cyclic element $E_{b}$ of $B$ there exists a unique true cyclic element $E_{a}$ of $A$ which maps onto $E_{b}$ topologically under $T$.

These are direct consequences of (2.6) and (2.7).

(3.3) The image of every true cyclic element $E_{a}$ of $A$ is either a single point, a true cyclic element $E_{b}$ of $B$, or a free arc of $B$ which is also a cyclic chain of $B$. If $T\left(E_{a}\right)=E_{b}$, then $T$ is topological on $E_{a}$.

Proof. We have at once that $T\left(E_{a}\right)$ is a cyclic chain $C(x, y)$ in $B$, if we assume that it is not a single point. If $C(x, y)$ is a single true cyclic element $E_{b}$ of $B$, then our conclusion follows at once. Hence we may assume that $x$ and $y$ are distinct and that there exists at least one point $z$ which separates $x$ and $y$ in $B$.

Let $x^{\prime}$ and $y^{\prime}$ be points of $E_{a}$ mapping into $x$ and $y$, respectively, and let $J$ be a simple closed curve in $E_{a}$ containing $x^{\prime}$ and $y^{\prime}$, and define $J^{\prime}=T(J)$. We first show that $J^{\prime}$ is a free arc of $B$. Regarding $J$ as our space, we see that $T$ is arc-preserving on $J$. If $J^{\prime}$ contains a true cyclic element $F$ of itself, then by (3.2), $T(J)=F$ is a homeomorphism. This is impossible since $x$ and $y$ are separated in $B$ by the point $z$. Thus $J^{\prime}$ is a dendrite. But $J^{\prime}$ contains no triod, since if it did we could easily find an arc of $J$ having a triod in its image. Therefore, $J^{\prime}$ is a simple arc.

Assume that $J^{\prime}=a^{\prime} d^{\prime} b^{\prime}$ is not a free arc of $B$. Then there exists a triod $t$ in $B$ having $d^{\prime}$ as center and $a^{\prime}, b^{\prime}$ as two of its end points, where $d^{\prime}$ is some interior point of $J^{\prime}$. Let $c^{\prime}$ be the other end point of $t$. Then the three arcs $a^{\prime} d^{\prime}, b^{\prime} d^{\prime}, c^{\prime} d^{\prime}$ of $t$ are disjoint except for $d^{\prime}$ and we may let $\left\{c_{i}^{\prime}\right\}$ be a sequence of distinct points on $c^{\prime} d^{\prime}$ converging to $d^{\prime}$ as a limit. It follows at once that there exists a point $d$ in $T^{-1}\left(d^{\prime}\right)$ and a sequence of points $\left\{c_{i}\right\}$ in $\left\{T^{-1}\left(c_{i}^{\prime}\right)\right\}$ converging to $d$.

Since $A$ is a locally connected continuum, there exists a region $R$ in $A$ 
containing $b$ but disjoint from both $T^{-1}\left(a^{\prime}\right)$ and $T^{-1}\left(b^{\prime}\right)$. This region is arcwise connected and hence contains a simple arc $c d$, where $c$ is one of the points $c_{i}$.

If $d$ lies on $J$ we let $z$ be the first intersection of $c d$ with $J$, and consider an arc $G$ defined as the sum of $c z$ and an arc of $J$ intersecting both $T^{-1}\left(a^{\prime}\right)$ and $T^{-1}\left(b^{\prime}\right)$. It is immediate that $T(G)$ contains a triod, which is impossible since $T$ is arc-preserving. Thus $d$ does not lie on $J$.

Let $z$ be the first intersection of the arc $c d$ with the closed set $T^{-1}\left(J^{\prime}\right)$, and define $c x e$ as a simple arc in $A$ having the unique point $e$ in common with $J$, and on $c x e$ let $y$ be the last intersection with $c z$. Define an arc $H$ as follows: $\left(\mathrm{a}^{\prime}\right)$ if $y$ is not $z$ then $H$ is the sum of the subarc $z y$ of $z c$ and the subarc $y e$ of $c x e$; $\left(\mathrm{b}^{\prime}\right)$ if $y$ is $z$ then $H$ is the sum of $c z$ and the subarc $z e$ of $c x e$. Let $G$ be a simple arc of $A$ composed of $H$ and a subarc of $J$ intersecting both $T^{-1}\left(a^{\prime}\right)$ and $T^{-1}\left(b^{\prime}\right)$. Then $T(G)$ contains a triod, which is impossible.

Thus $J^{\prime}$ is a free $\operatorname{arc} a^{\prime} b^{\prime}$ of $B$. Thus every point of the open subarc $x y$ of $a^{\prime} b^{\prime}$ must separate $x$ and $y$ in $B$. Accordingly, $C(x, y)=x y$ and $C(x, y)$ is a free arc of $B$.

(3.4) $\left(^{8}\right)$ If $a$ and $b$ are two points of $A$ having the same image point under $T$, then no true cyclic element of the chain $C(a, b)$ can map topologically under $T$. Thus each true cyclic element in $C(a, b)$ maps into either a single point or a free $\operatorname{arc}$ of $B$.

Proof. Let $T(a)=T(b)$ and suppose there is a cyclic element $E_{\alpha}$ of $C(a, b)$ which maps onto a cyclic element $E_{\beta}$ of $B$ topologically. Let aqrb be a simple arc in $A$ where $a q \cdot E_{\alpha}=q, r b \cdot E_{\alpha}=r$. Then either $a q$ or $r b$ is nondegenerate, and we may suppose $a q$ is nondegenerate ( $r b$ may or may not be nondegenerate). Then $T(a q+r b)=K$ is a continuum. Furthermore, $K \cdot E_{\beta}$ contains the two distinct points $T(q)$ and $T(r)$. Hence $K \cdot E_{\beta}$ contains a point $x$ distinct from both $T(q)$ and $T(r)$ which is the image of an internal point $x_{0}$ of $E_{\alpha}$. This is impossible, since $T^{-1}(x)$ also intersects $a q+r b-(q+r)$, whereas by (2.7) and (2.6), $T^{-1}(x)$ must consist of a single point.

4. Dendrite-preserving property of arc-preserving transformations. If $A$ is a dendrite (or tree) and $T(A)=B$ is arc-preserving, then it follows at once from (2.2) that $T$ is dendrite-preserving. This fact can also be seen from (2.5), since if $B$ had a true cyclic element $E_{b}$ then $A$ would also have one. This dendrite-preserving property of arc-preserving transformations was first noted and proven by R. G. Simond $\left({ }^{9}\right)$ as mentioned in the introduction of this paper. However, it is interesting to note that it follows directly from (2.4) of A.P.T. by the reasoning just given above, since the irreducibility of $T$

(8) This is closely related to (2.3) of A.P.T. and, indeed, yields (2.3) of A.P.T. as a special case.

$\left({ }^{9}\right)$ See footnote 3 . 
assumed in (2.4) of A.P.T. does not limit the generality because we can take a sub-dendrite of $A$ on which $T$ is irreducible.

In view of the considerable length and difficulty of Miss Simond's proof, the following one which is self-contained and independent of all other results on arc-preserving transformations may be of interest.

THEOREM (Simond). Arc-preserving transformations are dendrite-preserving.

Proof. Let $T(A)=B$ be arc-preserving, where $A$ is a dendrite. We first show:

I. (Whether $A$ is a dendrite or not.) If $t=o a+o b+o c$ is a triod such that $T(o)=o^{\prime}, T(a)=a^{\prime}, T(b)=b^{\prime}, T(c)=c^{\prime}$, and if $t \cdot T^{-1}\left(a^{\prime}+b^{\prime}+c^{\prime}\right)=a+b+c$, then $T(o a) \cdot T(o b)=T(o a) \cdot T(o c)=T(o b) \cdot T(o c)=T(o)=o^{\prime}$.

For if, say, $T(o a) \cdot T(o b)$ contains a point $q^{\prime}$ distinct from $o^{\prime}$, we may suppose the order $a^{\prime}, o^{\prime}, q^{\prime}, b^{\prime}$ on the arc $a^{\prime} o^{\prime} b^{\prime}=T(a o b)$. Then $T(a o)$ is a subarc $a^{\prime} o^{\prime} q^{\prime}$ of $a^{\prime} o^{\prime} b^{\prime}$. Hence the arc $T(a o c)$ consists of $a^{\prime} o^{\prime} q^{\prime}$ plus an arc $q^{\prime} c^{\prime}$ from $q^{\prime}$ to $c^{\prime}$ which contains neither $o^{\prime}$ nor $b^{\prime}$. But then the arc $T(b o c)=b^{\prime} o^{\prime} c^{\prime}$ would contain both the arc $o^{\prime} q^{\prime} b^{\prime}$ of $a^{\prime} o^{\prime} b^{\prime}$ and the arc $q^{\prime} c^{\prime}$, which is impossible since clearly $o^{\prime} q^{\prime} b^{\prime}+q^{\prime} c^{\prime}$ contains a triod. This proves $\mathrm{I}$.

Now suppose, contrary to the theorem, that $B$ has a true cyclic element $B^{\prime}$. Let $A^{\prime}$ be a minimal $A$-set in $A$ such that $T\left(A^{\prime}\right)$ contains $B^{\prime}$. Then since $A^{\prime}$ is a dendrite but not an arc, there exists a point $o$ in $A^{\prime}$ and three continua $X, Y, Z$ such that $A^{\prime}=X+Y+Z$ and $X \cdot Y=Y \cdot Z=Z \cdot X=o$. Let $T(o)=o^{\prime}$. Since $B^{\prime}$ is cyclic and $T(Y+Z)$ does not contain $B^{\prime}$, there exists a point $q^{\prime}$ in $B^{\prime}-o^{\prime}$ and points $x$ in $X, y$ in $(Y+Z)$, such that $T(x)=T(y)=q^{\prime}$. Clearly we may suppose $y$ in $Y$. Take the arcs $x o$ and $y o$ in $X$ and $Y$ respectively. Then since both $T(x o)$ and $Y(y o)$ contain $\operatorname{arcs}$ from $o^{\prime}$ to $q^{\prime}$ whereas $T(x o+o y)$ must be a simple arc, clearly $T(x o) \cdot T(o y)$ contains an arc from $o^{\prime}$ to $q^{\prime}$. Hence there is no loss of generality in assuming (as we shall do) that both $x$ and $y$ are cut points of $A^{\prime}$. Let $R_{x}$ and $R_{y}$ be components of $A^{\prime}-x$ and $A^{\prime}-y$ lying in $X-x$ and $Y-y$ respectively. Then since no one of the sets $T\left(A^{\prime}-R_{x}\right)$, $T\left(A^{\prime}-R_{y}\right), T(X+Y)$ contains $B^{\prime}$, there exist points $a^{\prime}, b^{\prime}$, and $c^{\prime}$ in $B^{\prime}$ such that $T^{-1}\left(a^{\prime}\right) \cdot A^{\prime} \subset R_{x}, T^{-1}\left(b^{\prime}\right) \cdot A^{\prime} \subset R_{y}, T^{-1}\left(c^{\prime}\right) \cdot A^{\prime} \subset Z-o$. Let $a \varepsilon T^{-1}\left(a^{\prime}\right)$, $b \varepsilon T^{-1}\left(b^{\prime}\right)$, and $c \varepsilon T^{-1}\left(c^{\prime}\right)$ be so chosen that for the arcs $a x, y b$, and $o c$ in $A^{\prime}$ we have $a x \cdot T^{-1}\left(a^{\prime}\right)=a, y b \cdot T^{-1}\left(b^{\prime}\right)=b$, and $T^{-1}\left(c^{\prime}\right) \cdot o c=c$. Let $o a=o x+x a$, $o b=o y+y b$. Then $t=o a+o b+o c$ is a triod satisfying the conditions in I. However, since each of the sets $T(o a)$ and $T(o b)$ contains both $o^{\prime}$ and $q^{\prime}$ we have a contradiction to I. Thus $B$ can have no true cyclic element and hence must be a dendrite.

5. $A$-set reversing transformations. In conclusion, we give a characterization of $A$-set reversing transformations which is made possible by (2.6) and (2.7). We recall that $T(A)=B$ is $A$-set reversing provided that for each $b$ 
in $B, T^{-1}(b)$ is either a single point or an $A$-set in $A$, it being assumed that $A$ is a compact locally connected continuum. We make use here of certain results concerning this type of transformation which were established in $\S 4$ of A.P.T.

Theorem. If $A$ is a compact locally connected continuum and $T(A)=B$ is arc-preserving, then in order that $T$ be $A$-set reversing it is necessary and suffcient that the following conditions hold: (a) there exists no true cyclic element $E$ in $A$ such that $T(E)$ is a free arc of $B$; (b) if $K$ is the set of all cut points and end points of $A$, then $T$ is monotone on $T(K)$.

Proof. The necessity follows at once from the definition and the fact that every $A$-set reversing transformation is monotone (A.P.T., (4.12)).

Sufficiency: By (A.P.T., (4.1)) we must show that $T$ is monotone on each simple $\operatorname{arc}$ in $A$. If this is not the case, there exists a simple arc $a x b$ in $A$ such that $T(a)=T(b) \neq T(x)$ for any point $x$ interior to $a x b$. If $a$ and $b$ are conjugate points, they lie in the same true cyclic element $E$ of $A$ and it is immediate from (a) and (3.3) that $T$ is monotone on $a x b$. Thus there exists a point $q$ interior to $a x b$ which separates $a$ and $b$ in $A$. It follows at once from (b) that $a$ is an internal point of a true cyclic element $E$ of $A$ which maps topologically on to a true cyclic element $F$ of $B$. But $T^{-1}$ is single valued on $T\left(E^{0}\right)$, where $E^{0}$ represents the set of all internal points of $E$.

BRown UNIVERSITY,

Providence, R. I.,

The University of Virginia,

Charlottesville, Va. 